\title{
On hamiltonian colorings of block graphs
}

\author{
Devsi Bantva \\ Lukhdhirji Engineering College, Morvi - 363642 \\ Gujarat (INDIA) \\ E-mail:devsi.bantva@gmail.com
}

\begin{abstract}
A hamiltonian coloring $c$ of a graph $G$ of order $p$ is an assignment of colors to the vertices of $G$ such that $D(u, v)+|c(u)-c(v)| \geq$ $p-1$ for every two distinct vertices $u$ and $v$ of $G$, where $D(u, v)$ denotes the detour distance between $u$ and $v$. The value $h c(c)$ of a hamiltonian coloring $c$ is the maximum color assigned to a vertex of $G$. The hamiltonian chromatic number, denoted by $h c(G)$, is the $\min \{h c(c)\}$ taken over all hamiltonian coloring $c$ of $G$. In this paper, we present a lower bound for the hamiltonian chromatic number of block graphs and give a sufficient condition to achieve the lower bound. We characterize symmetric block graphs achieving this lower bound. We present two algorithms for optimal hamiltonian coloring of symmetric block graphs.
\end{abstract}

Keywords: Hamiltonian coloring, hamiltonian chromatic number, block graph, symmetric block graph.

\section{Introduction}

A hamiltonian coloring $c$ of a graph $G$ of order $p$ is an assignment of colors (nonnegative integers) to the vertices of $G$ such that $D(u, v)+|c(u)-c(v)| \geq p-1$ for every two distinct vertices $u$ and $v$ of $G$, where $D(u, v)$ denotes the detour distance which is the length of the longest path between $u$ and $v$. The value of $h c(c)$ of a hamiltonian coloring $c$ is the maximum color assigned to a vertex of $G$. The hamiltonian chromatic number $h c(G)$ of $G$ is $\min \{h c(c)\}$ taken over all hamiltonian coloring $c$ of $G$. It is clear from definition that two vertices $u$ and $v$ can be assigned the same color only if $G$ contains a hamiltonian $u-v$ path. Moreover, if $G$ is a hamiltonian-connected graph then all the vertices can be assigned the same color. Thus the hamiltonian chromatic number of a connected graph $G$ measures how close $G$ is to being hamiltonian-connected, minimum the hamiltonian chromatic number of a connected graph $G$ is, the closer $G$ is to being hamiltonian-connected. The concept of hamiltonian coloring was introduced by Chartrand et al. 2] as a variation of radio k-coloring of graphs.

At present, the hamiltonian chromatic number is known only for handful of graph families. Chartrand et al. investigated the exact hamiltonian chromatic numbers for complete graph $K_{n}$, cycle $C_{n}$, star $K_{1, n}$ and complete bipartite graph $K_{r, s}$ in [2]3. Also an upper bound for $h c\left(P_{n}\right)$ was established by Chartrand et al. in 2] but the exact value of $h c\left(P_{n}\right)$ which is equal to the radio antipodal 
number $a c\left(P_{n}\right)$ given by Khennoufa and Togni in [5]. In [6], Shen et al. have discussed the hamiltonian chromatic number for graphs $G$ with $\max \{D(u, v)$ : $u, v \in V(G), u \neq v\} \leq \frac{p}{2}$, where $p$ is the order of graph $G$ and they gave the hamiltonian chromatic number for a special class of caterpillars and double stars. The researchers emphasize that determining the hamiltonian chromatic number is interesting but a challenging task even for some basic graph families.

Without loss of generality, we initiate with label 0 , then the span of any hamiltonian coloring $c$ which is defined as $\max \{|c(u)-c(v)|: u, v \in V(G)\}$, is the maximum integer used for coloring. However, in [2]3[6] only positive integers are used as colors. Therefore, the hamiltonian chromatic number defined in this article is one less than that defined in [2]36] and hence we will make necessary adjustment when we present the results of [236] in this article. Moreover, for standard graph theoretic terminology and notation we follow [8].

In this paper, we present a lower bound for the hamiltonian chromatic number of block graphs and give a sufficient condition to achieve the lower bound. As an illustration, we present symmetric block graphs (those block graphs whose all blocks are cliques of size $n$, each cut vertex is exactly in $k$ blocks and the eccentricity of end vertices is same) achieving this lower bound. We present two algorithms for optimal hamiltonian coloring of symmetric block graphs.

\section{A lower bound for hamiltonian chromatic number of block graphs}

A block graph is a connected graph all of whose blocks are cliques. The detour distance between $u$ and $v$, denoted by $D(u, v)$, is the longest distance between $u$ and $v$ in $G$. The detour eccentricity $\epsilon_{D}(v)$ of a vertex $v$ is the detour distance from $v$ to a vertex farthest from $v$. The detour center $C_{D}(G)$ of $G$ is the subgraph of $G$ induced by the vertex/vertices of $G$ whose detour eccentricity is minimum. In 4, Chartrand et al. shown that the detour center $C_{D}(G)$ of every connected graph $G$ lies in a single block of $G$. The vertex/vertices of detour center $C_{D}(G)$ are called detour central vertex/vertices for graph $G$. In a block graph $G$, if $u$ is on the $w-v$ path, where $w$ is the nearest detour central vertex for $v$, then $u$ is an ancestor of $v$, and $v$ is a descendent of $u$. Let $u_{i}, i=1,2, \ldots, n$ are adjacent vertices of a block attached to a central vertex. Then the subgraph induced by $u_{i}, i=1,2, \ldots, n$ and all its descendent is called a branch at $w$. Two branches are called different if they are induced by vertices of two different blocks attached to the same central vertex, and opposite if they are induced by vertices of two different blocks attached to different central vertices. For a block graph $G$, define detour level function $\mathcal{L}$ on $V(G)$ by

$$
\mathcal{L}(u):=\min \left\{D(w, u): w \in V\left(C_{D}(G)\right)\right\} \text {, for any } u \in V(G) .
$$

The total detour level of a graph $G$, denoted by $\mathcal{L}(G)$, is defined as

$$
\mathcal{L}(G):=\sum_{u \in V(G)} \mathcal{L}(u)
$$


Note that if $\left|C_{D}(G)\right|=\omega$ then the detour distance between any two vertices $u$ and $v$ in a block graph $G$ satisfies

$$
D(u, v) \leq \mathcal{L}(u)+\mathcal{L}(v)+\omega-1 .
$$

Moreover, equality holds in (22) if $u$ and $v$ are in different branches when $\omega$ $=1$ and in opposite branches when $\omega \geq 2$.

Define $\xi=\min \left\{\left|V\left(B_{i}\right)\right|-1: B_{i}\right.$ is a block attached to detour central vertex $\}$ when $\omega=1$; otherwise $\xi=0$.

We first give a lower bound for the hamiltonian chromatic number of block graphs. A hamiltonian coloring $c$ on $V(G)$, induces an ordering of $V(G)$, which is a line up of the vertices with equal or increasing images. We denote this ordering by $V(G)=\left\{u_{0}, u_{1}, u_{2}, \ldots, u_{p-1}\right\}$ with

$0=c\left(u_{0}\right) \leq c\left(u_{1}\right) \leq c\left(u_{2}\right) \leq \ldots \leq c\left(u_{p-1}\right)$.

Notice that, $c$ is a hamiltonian coloring, then the span of $c$ is $c\left(u_{p-1}\right)$.

Theorem 1. Let $G$ be a block graph of order $p$ and $\omega, \xi$ and $\mathcal{L}(G)$ are defined as earlier then

$$
h c(G) \geq(p-1)(p-\omega)-2 \mathcal{L}(G)+\xi .
$$

Proof. It suffices to prove that any hamiltonian coloring $c$ of block graph $G$ has no span less than the right hand side of (3). Let $c$ be an arbitrary hamiltonian coloring for $G$, where $0=c\left(u_{0}\right) \leq c\left(u_{1}\right) \leq c\left(u_{2}\right) \leq \ldots \leq c\left(u_{p-1}\right)$. Then $c\left(u_{i+1}\right)-$ $c\left(u_{i}\right) \geq p-1-D\left(u_{i}, u_{i+1}\right)$, for all $0 \leq i \leq p-2$. Summing up these $p-1$ inequalities, we get

$$
c\left(u_{p-1}\right)-c\left(u_{0}\right) \geq(p-1)^{2}-\sum_{i=0}^{p-1} D\left(u_{i}, u_{i+1}\right)
$$

We consider following two cases.

Case-1: $\omega=1$. In this case, note that $\mathcal{L}\left(u_{0}\right)+\mathcal{L}\left(u_{p-1}\right) \geq \xi$ and by substituting (2) into (4) we obtain,

$$
\begin{aligned}
c\left(u_{p-1}\right)-c\left(u_{0}\right) & \geq(p-1)^{2}-\sum_{i=0}^{p-1} D\left(u_{i}, u_{i+1}\right) \\
& \geq(p-1)^{2}-\sum_{i=0}^{p-1}\left(\mathcal{L}\left(u_{i}\right)+\mathcal{L}\left(u_{i+1}\right)+\omega-1\right) \\
& =(p-1)^{2}-2 \sum_{u \in V(G)} \mathcal{L}(u)+\mathcal{L}\left(u_{0}\right)+\mathcal{L}\left(u_{p-1}\right)-(p-1)(\omega-1) \\
& \geq(p-1)(p-\omega)-2 \mathcal{L}(G)+\xi
\end{aligned}
$$


Case-2: $\omega \geq 2$. In this case, note that $\mathcal{L}\left(u_{0}\right)+\mathcal{L}\left(u_{p-1}\right) \geq 0$ and by substituting (21) into (41) we obtain,

$$
\begin{aligned}
c\left(u_{p-1}\right)-c\left(u_{0}\right) & \geq(p-1)^{2}-\sum_{i=0}^{p-1} D\left(u_{i}, u_{i+1}\right) \\
& \geq(p-1)^{2}-\sum_{i=0}^{p-1}\left(\mathcal{L}\left(u_{i}\right)+\mathcal{L}\left(u_{i+1}\right)+\omega-1\right) \\
& =(p-1)^{2}-2 \sum_{u \in V(G)} \mathcal{L}(u)+\mathcal{L}\left(u_{0}\right)+\mathcal{L}\left(u_{p-1}\right)-(p-1)(\omega-1) \\
& \geq(p-1)(p-\omega)-2 \mathcal{L}(G) \\
& =(p-1)(p-\omega)-2 \mathcal{L}(G)+\xi
\end{aligned}
$$

Thus, any hamiltonian coloring has span not less than the right hand side of (3) and hence we obtain $h c(G) \geq(p-1)(p-\omega)-2 \mathcal{L}(G)+\xi$.

The next result gives sufficient condition with optimal hamiltonian coloring for the equality in (3).

Theorem 2. Let $G$ be a block graph of order $p$ and, $\omega, \xi$ and $\mathcal{L}(G)$ are defined as earlier then

$$
h c(G)=(p-1)(p-\omega)-2 \mathcal{L}(G)+\xi,
$$

if there exists an ordering $\left\{u_{0}, u_{1}, \ldots, u_{p-1}\right\}$ with $0=c\left(u_{0}\right) \leq c\left(u_{1}\right) \leq \ldots \leq$ $c\left(u_{p-1}\right)$ of vertices of block graph $G$ such that

1. $\mathcal{L}\left(u_{0}\right)=0, \mathcal{L}\left(u_{p-1}\right)=\xi$ when $\omega=1$ and $\mathcal{L}\left(u_{0}\right)=\mathcal{L}\left(u_{p-1}\right)=0$ when $\omega \geq 2$,

2. $u_{i}$ and $u_{i+1}$ are in different branches when $\omega=1$ and opposite branches when $\omega \geq 2$,

3. $D\left(u_{i}, u_{i+1}\right) \leq \frac{p}{2}$, for $0 \leq i \leq p-2$.

Moreover, under these conditions the mapping $c$ defined by

$$
\begin{gathered}
c\left(u_{0}\right)=0 \\
c\left(u_{i+1}\right)=c\left(u_{i}\right)+p-1-\mathcal{L}\left(u_{i}\right)-\mathcal{L}\left(u_{i+1}\right)-\omega+1,0 \leq i \leq p-2
\end{gathered}
$$

is an optimal hamiltonian coloring of $G$.

Proof. Suppose (1), (2) and (3) hold for an ordering $\left\{u_{0}, u_{1}, \ldots, u_{p-1}\right\}$ of the vertices of $G$ and $c$ is defined by (6) and (7). By Theorem 10 it is enough to prove that $c$ is a hamiltonian coloring whose span is $c\left(u_{p-1}\right)=(p-1)(p-\omega)-2 \mathcal{L}(G)$ $+\xi$. 
Without loss of generality assume that $j-i \geq 2$ then

$$
\begin{aligned}
c\left(u_{j}\right)-c\left(u_{i}\right) & =\sum_{\substack{t=i \\
j-1}}^{j-1}\left[c\left(u_{t+1}\right)-c\left(u_{t}\right)\right] \\
& \geq \sum_{\substack{t=i \\
j-1}}^{j-1}\left[p-1-\mathcal{L}\left(u_{t}\right)-\mathcal{L}\left(u_{t+1}\right)-w+1\right] \\
& \geq \sum_{t=i}\left[p-1-D\left(u_{t}, u_{t+1}\right)\right] \\
& =(j-i)(p-1)-\sum_{t=i}^{j-1} D\left(u_{t}, u_{t+1}\right) \\
& \geq(j-i)(p-1)-(j-i)\left(\frac{p}{2}\right) \\
& =(j-i)\left(\frac{p-1}{2}\right) \\
& =p-2
\end{aligned}
$$

Note that $D\left(u_{i}, u_{i+1}\right) \geq 1$; it follows that $\left|c\left(u_{j}\right)-c\left(u_{i}\right)\right|+D\left(u_{i}, u_{i+1}\right) \geq p-1$. Hence, $c$ is a hamiltonian coloring for $G$. The span of $c$ is given by

$$
\begin{aligned}
\operatorname{span}(c) & =\sum_{t=0}^{p-2}\left[c\left(u_{t+1}\right)-c\left(u_{t}\right)\right] \\
& =\sum_{t=0}^{p-2}\left[p-1-\mathcal{L}\left(u_{t}\right)-\mathcal{L}\left(u_{t+1}\right)-\omega+1\right] \\
& =(p-1)^{2}-\sum_{t=0}^{p-2}\left[\mathcal{L}\left(u_{t}\right)+\mathcal{L}\left(u_{t+1}\right)\right]-(p-1)(\omega-1) \\
& =(p-1)(p-\omega)-2 \sum_{u \in V(G)} \mathcal{L}(u)+\mathcal{L}\left(u_{0}\right)+\mathcal{L}\left(u_{p-1}\right) \\
& =(p-1)(p-\omega)-2 \mathcal{L}(G)+\xi
\end{aligned}
$$

Therefore, $h c(G) \leq(p-1)(p-\omega)-2 \mathcal{L}(G)+\xi$. This together with (3) implies (5) and that $c$ is an optimal hamiltonian coloring.

\section{Hamiltonian chromatic number of symmetric block graphs}

In this section, we continue to use the terminology and notation defined in previous section. We use Theorem 1 and 2 to determine the hamiltonian chromatic number of symmetric block graphs.

A symmetric block graph, denoted by $B_{n, k}\left(\right.$ or $B_{n, k}(d)$ if diameter is $\left.d\right)$, is a block graph with at least two blocks such that all blocks are cliques of size $n$, each cut vertex is exactly in $k$ blocks and the eccentricity of end vertices is same 
(see Figure 1). It is straight forward to verify that the detour center of symmetric block graph of diameter $d$ is a vertex when $d$ is even and a block of size $n$ when $d$ is odd. Consequently, the number of detour central vertex/vertices for a symmetric block graph $B_{n, k}$ of diameter $d$ is either 1 or $n$ depending upon $d$ is even or odd. We observe that $B_{2, k}(2)$ are stars $K_{1, k}, B_{n, k}(2)$ are one point union of $k$ complete graphs (a one point union of $k$ complete graphs, also denoted by $K_{n}^{k}$, is a graph obtained by taking $v$ as a common vertex such that any two copies of $K_{n}$ are edge disjoint and do not have any vertex common except $v), B_{2,2}(d)$ are paths $P_{d+1}$ and $B_{2, k}(d)$ are symmetric trees (see 7]). The hamiltonian chromatic number of stars $K_{1, k}$ is reported by Chartrand et al. in 2. The hamiltonian chromatic number of paths which is equal to the antipodal radio number of paths given by Khennoufa and Togni in $[5$ and the hamiltonian chromatic number of symmetric trees is investigated by Bantva in [1]. Hence we consider $k \geq 2$ and $d, n \geq 3$. However, for completeness we first give the hamiltonian chromatic number for $B_{n, k}(2)$ in Theorem 6 and next we consider general case.

Theorem 3. [Q] For $n \geq 3, h c\left(K_{1, n}\right)=(n-1)^{2}$.

Theorem 4. [5] For any $n \geq 5$,

$$
h c\left(P_{n}\right)=a c\left(P_{n}\right)=\left\{\begin{array}{l}
2 p^{2}-2 p+2, \text { if } n=2 p+1, \\
2 p^{2}-4 p+4, \text { if } n=2 p .
\end{array}\right.
$$

Theorem 5. [1] Let $T$ be a symmetric tree of order $p \geq 4$ and $\Delta(T) \geq 3$. Then

$$
h c(T)=(p-1)(p-1-\epsilon(T))+\epsilon^{\prime}(T)-2 \mathcal{L}(T),
$$

where $\epsilon(T)=0$ when $C(T)=\{w\}$ and $\epsilon(T)=1$ when $C(T)=\left\{w, w^{\prime}\right\}$; and $\epsilon^{\prime}(T)=1-\epsilon(T)$

The next result gives the hamiltonian chromatic number for one point union of $k$ copies of complete graph $K_{n}$.

Theorem 6. For $n, k \geq 2$,

$$
h c\left(K_{n}^{k}\right)= \begin{cases}(n-1)^{2}, & \text { if } k=2, \\ k(k-2)(n-1)^{2}+n-1, & \text { if } k \geq 3 .\end{cases}
$$

Proof. Let $K_{n}^{k}$ be one point union of $k$ complete graph. To prove the result we consider following two cases.

Case - 1: $k=2$. Let $G=K_{n}^{2}$ with vertex set $\left\{x_{1}, x_{2}, \ldots, x_{n-1}, y_{1}, y_{2}, \ldots\right.$, $\left.y_{n-1}, z\right\}$, where $x_{i}$ and $y_{i}, 1 \leq i \leq n-1$ be the vertices of block on each side and $z$ is the common vertex of two blocks in $G$. Let $c$ be a minimum hamiltonian coloring of $G$ with $0 \in c(V(G))$. Since $G$ contains hamiltonian path between $x_{i}$ and $y_{i}$ for $1 \leq i \leq n-1$, we can color $x_{i}$ and $y_{i}$ with same color. Since $D\left(z, x_{i}\right)=D\left(z, y_{i}\right)=D\left(x_{i}, x_{j}\right)=D\left(y_{i}, y_{j}\right)=n-1$ and $D\left(x_{i}, y_{j}\right)=2 n-2=$ $p-1$, for $1 \leq i, j \leq n-1$ and $i \neq j$. It follows that $\left|c(z)-c\left(x_{j}\right)\right| \geq n-1$ and 
$\left|c\left(x_{i}\right)-c\left(x_{j}\right)\right| \geq n-1$. This implies that $h c(G)=h c(c) \geq 0+(n-1)(n-1)=$ $(n-1)^{2}$.

Next we show that $h c(G) \leq(n-1)^{2}$. To prove this, it is enough to give hamiltonian coloring with span equal to $(n-1)^{2}$. Define a coloring $c$ of $G$ by

$$
\begin{aligned}
& c(z)=0 \\
& c\left(x_{i}\right)=c\left(y_{i}\right)=i(n-1), 1 \leq i \leq n-1
\end{aligned}
$$

Since $c$ is a hamiltonian coloring, $h c(G) \leq h c(c)=c\left(x_{n-1}\right)=c\left(y_{n-1}\right)=$ $(n-1)(n-1)=(n-1)^{2}$ and hence $h c(G)=h c\left(K_{n}^{(2)}\right)=(n-1)^{2}$.

Case - 2: $k \geq 3$. Let $G=K_{n}^{k}$ with vertex set $\left\{v_{i}^{j}, w: 1 \leq i \leq n-1,1 \leq j \leq\right.$ $k\}$ such that for each $j=1,2, \ldots, k, v_{i}^{j}$ where $1 \leq i \leq n-1$ are in same block and $w$ is the common vertex of $G$. Define a coloring $c$ of $G$ by

$$
\begin{aligned}
& c(w)=0 \\
& c\left(v_{1}^{1}\right)=(k-1)(n-1) \\
& c\left(v_{i+1}^{1}\right)=c\left(v_{i}^{1}\right)+k(n-1), 2 \leq i \leq n-1 \\
& \text { For } j=1,2, \ldots, k-1 \\
& c\left(v_{i}^{j+1}\right)=c\left(v_{i}^{j}\right)+(k-2)(n-1), 1 \leq i \leq n-1 .
\end{aligned}
$$

Since $c$ is a hamiltonian coloring, $h c(G) \leq h c(c)=(k-1)(n-1)+(k-1)(k-$ $2)(n-1)+k(k-2)(n-1)(n-2)=k(k-2)(n-1)^{2}+n-1$.

Now we show that $h c(G) \geq k(k-2)(n-1)^{2}+n-1$. Let $c$ be a minimum hamiltonian coloring of $G$. Since $G$ contains no hamiltonian path no two vertices can be colored the same. A hamiltonian coloring induces an ordering on $V(G)$ with increasing images. We may assume that $0=c\left(u_{0}\right)<c\left(u_{1}\right)<\ldots<c\left(u_{p-1}\right)$. We consider three subcases.

Subcase - 1: $c(w)=c\left(u_{0}\right)=0$. Since $D\left(u_{0}, u_{1}\right)=D\left(w, u_{1}\right)=n-1$, $c\left(u_{1}\right) \geq(k-1)(n-1)$. Also $D\left(v_{x}^{j}, v_{y}^{j}\right)=n-1$ for $1 \leq j \leq k, x \neq y$ and $D\left(v_{x}^{t}, v_{y}^{l}\right)$ for $1 \leq x, y \leq n-1,1 \leq t, l(t \neq l) \leq k$. It follows that $c\left(u_{3}\right) \geq(k-2)(n-1)$ and $c\left(u_{i+1}\right) \geq c\left(u_{i}\right)+(k-2)(n-1)$ for all $3 \leq i \leq k(n-1)-1$. This implies that $c\left(u_{p-1}\right) \geq(k-1)(n-1)+(k(n-1)-1)(k-2)(n-1)=k(k-2)(n-1)^{2}+n-1$. Therefore $h c(c) \geq k(k-2)(n-1)^{2}+n-1$.

Subcase - 2: $c(w)=c\left(u_{p-1}\right)=h c(c)$. Since $D\left(v_{x}^{j}, v_{y}^{j}\right)=n-1$ for $1 \leq j \leq k$, $x \neq y$ and $D\left(v_{x}^{t}, v_{y}^{l}\right)$ for $1 \leq x, y \leq n-1,1 \leq t, l(t \neq l) \leq k$. For each $i$ with $1 \leq i \leq k(n-1)-1, c\left(v_{1}\right)=0$ and $c\left(v_{i+1}\right)=(k-2)(n-1)$ and $c(w)=$ $c\left(u_{p-1}\right)=c\left(u_{k(n-1)-1}\right)+(k-1)(n-1)$. This implies that $c\left(u_{p-1}\right)=c(w) \geq$ $(k(n-1)-1)(k-2)(n-1)+(k-1)(n-1)=k(k-2)(n-1)^{2}+n-1$. Therefore $h c(c) \geq k(k-2)(n-1)^{2}+n-1$.

Subcase - 3: $c\left(u_{i}\right) \leq c(w) \leq c\left(u_{i+1}\right)$ for some $i$ with $1 \leq i \leq k(n-1)-$ 1. Since $D\left(v_{x}^{j}, v_{y}^{j}\right)=n-1$ for $1 \leq j \leq k, x \neq y$ and $D\left(v_{x}^{t}, v_{y}^{l}\right)=n-2$ for $1 \leq x, y \leq n-1,1 \leq t, l(t \neq l) \leq k$. Define $c\left(u_{0}\right)=0$ and $c\left(u_{i+1}\right)=$ $c\left(u_{i}\right)+(k-2)(n-1)$ for $1 \leq i \leq m$ and $m \leq p-3$. Then $c\left(u_{m+1}\right)=c(w)$ $=c\left(u_{m}\right)+(k-1)(n-1)$ and $c\left(u_{m+2}\right)=c\left(u_{m+1}\right)+(k-1)(n-1), c\left(u_{i+1}\right)=$ $c\left(u_{i}\right)+(k-2)(n-1)$ for $m+2 \leq i \leq p-1$. Therefore $h c(c) \geq k(k-2)(n-$ $1)^{2}+2(n-1)$.

Hence from Subcase - 1, 2 and $3, h c(G)=k(k-2)(n-1)^{2}+n-1$. 
Thus, from Case - 1 and 2, we have

$$
h c\left(K_{n}^{k}\right)= \begin{cases}(n-1)^{2}, & \text { if } k=2, \\ k(k-2)(n-1)^{2}+n-1, & \text { if } k \geq 3 .\end{cases}
$$

We now determine the hamiltonian chromatics number for $B_{n, k}(d)$ for $k \geq 2$, $n, d \geq 3$ using Theorem 2, Note that $B_{3,2}(3), B_{3,3}(3)$ and $B_{3,2}(4)$ block graphs does not satisfies condition (c) of Theorem 2 but it is easy to verify that the hamiltonian chromatic numbers for these three graphs are coincide with the numbers produce by the formula stated in Theorem 7 . Moreover, labels assigned by Algorithms given in proof of Theorem 7 is the optimal hamiltonian coloring for these graphs.

Theorem 7. Let $k \geq 1, n \geq 2, d \geq 3$ be integers, $r=\left\lfloor\frac{d}{2}\right\rfloor$ and $\Phi_{r}(x)=$ $1+x+x^{2}+\ldots+x^{r-1}$. Then $h c\left(B_{n+1, k+1}(d)\right)$

$$
=\left\{\begin{array}{l}
n^{2}(k+1)\left[\Phi_{r}(k n)\left((k+1) \Phi_{r}(k n)-2 r\right)+\frac{2\left(\Phi_{r}(k n)-r\right)}{k n-1}\right]+n, \quad \text { if } d \text { is even, } \\
k n^{2}(n+1)\left[\Phi_{r}(k n)\left(k(n+1) \Phi_{r}(k n)-2 r+1\right)+\frac{2\left(\Phi_{r}(k n)-r\right)}{k n-1}\right],
\end{array}\right.
$$

Proof. The order $p$ and total detour level of $B_{n+1, k+1}(d)$ is given by

$$
\begin{gathered}
p=\left\{\begin{array}{l}
1+\sum_{i=1}^{r}(k+1) k^{i-1} n^{i}, \text { if } d \text { is even, } \\
1+n+\sum_{i=1}^{r} k^{i} n^{i+1}, \quad \text { if } d \text { is odd. }
\end{array}\right. \\
\mathcal{L}(G)= \begin{cases}n^{2}(k+1)\left(r \Phi_{r}(k n)+\frac{r-\Phi_{r}(k n)}{k n-1}\right), & \text { if } d \text { is even, } \\
k n^{2}(n+1)\left(r \Phi_{r}(k n)+\frac{r-\Phi_{r}(k n)}{k n-1}\right), & \text { if } d \text { is odd } .\end{cases}
\end{gathered}
$$

Substituting (9) and (10) into (3) gives the right hand side of (8).

We now prove that the right hand side of (8) is the actual value for the hamiltonian chromatics number of symmetric block graph. For this purpose we give a systematic hamiltonian coloring whose span is the right hand side of (8). We consider following two cases.

Case - 1: $d$ is even. In this case, symmetric block graphs has only one detour central vertex say $w$. We apply the following algorithm to find a hamiltonian coloring of symmetric block graph of even diameter whose span is right-hand side of (8).

Algorithm 1: An optimal hamiltonian coloring of symmetric block graphs $B_{n+1, k+1}(d)$, where $d$ is even.

Input: A symmetric block graph $B_{n+1, k+1}$ of even diameter. 
Idea: Find an ordering of vertices of block graphs $B_{n+1, k+1}$ of even diameter which satisfies Theorem 2 and labeling defined by (6)-(7) is a hamiltonian coloring whose span is right-hand side of (8).

Initialization: Start with a central vertex $w$.

Iteration: Define $c: V\left(B_{n+1, k+1}\right) \rightarrow\{0,1,2, .$.$\} as follows:$

Step-1: Let $v^{1}, v^{2}, \ldots, v^{(k+1) n}$ be the vertices adjacent to $w$ such that any $k+1$ consecutive vertices in the list are in different blocks.

Step-2: Now $k n$ descendent vertices of each $v^{t}, t=1,2, \ldots,(k+1) n$ by $v_{0}^{t}$, $v_{1}^{t}, \ldots, v_{k n-1}^{t}$ such that any $k$ consecutive vertices in the list are in different blocks. Next the $k n$ descendent vertices of each $v_{l}^{t}, 0 \leq l \leq k n-1,1 \leq t \leq(k+1) n$ by $v_{l 0}^{t}, v_{l 1}^{t}, \ldots, v_{l(k n-1)}^{t}$ such that any $k$ consecutive vertices lies in different blocks; inductively $k n$ descendent vertices of $v_{i_{1}, i_{2}, \ldots, i_{l}}^{t}\left(0 \leq i_{1}, i_{2}, \ldots, i_{l} \leq k n-1\right.$, $1 \leq t \leq(k+1) n)$ are indexed by $v_{i_{1}, i_{2}, \ldots, i_{l}, i_{l+1}}^{t}$ where $i_{l+1}=0,1, \ldots, k n-1$ such that any $k$ consecutive vertices in the list are in different blocks.

Step-3: Rename $v_{i_{1}, i_{2}, \ldots, i_{l}, i_{l+1}}^{t}$ by $v_{j}^{t},(1 \leq t \leq(k+1) n)$, where

$$
j=1+i_{1}+i_{2}(k n)+\ldots+i_{l}(k n)^{l-1}+\sum_{l+1 \leq t \leq \frac{d}{2}}(k n)^{t} .
$$

Step-4: Give ordering $\left\{u_{0}, u_{1}, \ldots, u_{p-1}\right\}$ of vertices of symmetric block graphs as follows.

For $1 \leq j \leq p-(k+1) n-1$, let

$u_{j}:=\left\{\begin{array}{l}v_{s}^{t}, \text { where } s=\left\lceil\frac{j}{(k+1) n}\right\rceil, \text { if } j \equiv t(\bmod (k+1) n), 1 \leq t \leq(k+1) n-1, \\ v_{s}^{(k+1) n}, \text { where } s=\left\lceil\frac{j}{(k+1) n}\right\rceil, \text { if } j \equiv 0(\bmod (k+1) n)\end{array}\right.$

For $p-(k+1) n \leq j \leq p-1$, let

$$
u_{j}:=v^{j-p+(k+1) n+1} .
$$

Then above defined ordering $\left\{u_{0}, u_{1}, \ldots, u_{p-1}\right\}$ of vertices satisfies Theorem 2 Step-5: Define $c: V\left(B_{n+1, k+1}\right) \rightarrow\{0,1,2, \ldots\}$ by $c\left(u_{0}\right)=0$ and $c\left(u_{i+1}\right)=$ $c\left(u_{i}\right)+p-1-\mathcal{L}\left(u_{i}\right)-\mathcal{L}\left(u_{i+1}\right)-\omega+1,0 \leq i \leq p-2$.

Output: The span of $c$ is $\operatorname{span}(c)=c\left(u_{p-1}\right)=c\left(u_{0}\right)+(p-1)^{2}-2\left(\sum_{u \in V(G)} \mathcal{L}(u)\right)+$ $n-1=(p-1)^{2}-2 \mathcal{L}\left(B_{n+1, k+1}\right)+n-1$ which is exactly the right-hand side of (8) by using (9) and (10) in the case of symmetric block graphs.

Case - 2: $d$ is odd. In this case, symmetric block graphs has $n+1$ central vertices say $v^{1}, v^{2}, \ldots, v^{n+1}$. We apply the following algorithm to find a hamiltonian coloring of symmetric block graph of odd diameter whose span is right-hand side (8).

Algorithm 2: An optimal hamiltonian coloring of symmetric block graphs $B_{n+1, k+1}(d)$, where $d$ is odd.

Input: A symmetric block graph $B_{n+1, k+1}(d)$ of odd diameter $d$.

Idea: Find an ordering of vertices of block graphs $B_{n+1, k+1}$ of odd diameter 
which satisfies Theorem 2 and labeling defined by (6)-(7) is a hamiltonian coloring whose span is right-hand side of (8).

Initialization: Starts with central vertices $v^{1}, v^{2}, \ldots, v^{n+1}$.

Iteration: Define $c: V\left(B_{n+1, k+1}\right) \rightarrow\{0,1,2, \ldots\}$

Step-1: Now $k n$ descendent vertices of each $v^{t}, t=1,2, \ldots,(k+1) n$ by $v_{0}^{t}$, $v_{1}^{t}, \ldots, v_{k n-1}^{t}$ such that any $k$ consecutive vertices in the list are in different blocks. Next $k n$ descendent vertices of each $v_{l}^{t}, 0 \leq l \leq k n-1,1 \leq t \leq n+1$ by $v_{l 0}^{t}$, $v_{l 1}^{t}, \ldots, v_{l(k n-1)}^{t}$ such that any $k$ consecutive vertices are in different blocks; inductively $k n$ descendent vertices of $v_{i_{1}, i_{2}, \ldots, i_{l}}^{t}\left(0 \leq i_{1}, i_{2}, \ldots, i_{l} \leq k n-1,1 \leq t \leq n+1\right)$ are indexed by $v_{i_{1}, i_{2}, \ldots, i_{l}, i_{l+1}}^{t}$ where $i_{l+1}=0,1, \ldots, k n-1$ such that any $k$ consecutive vertices in the list are in different blocks.

Step-2: We rename $v_{i_{1}, i_{2}, \ldots, i_{l}, i_{l+1}}^{t}$ by $v_{j}^{t},(1 \leq t \leq n+1)$, where

$$
j=1+i_{1}+i_{2}(k n)+\ldots+i_{l}(k n)^{l-1}+\sum_{l+1 \leq t \leq \frac{d-1}{2}}(k n)^{t} .
$$

Step-3: Define an ordering $\left\{u_{0}, u_{1}, \ldots, u_{p-1}\right\}$ as follows:

For $1 \leq j \leq p-n-1$, let

$$
u_{j}:=\left\{\begin{array}{l}
v_{s}^{t}, \text { where } s=\lceil j /(n+1)\rceil, \text { if } j \equiv t(\bmod (n+1)) \text { with } 1 \leq t \leq n, \\
v_{s}^{n+1}, \text { where } s=\lceil j /(n+1)\rceil, \text { if } j \equiv 0(\bmod (n+1))
\end{array}\right.
$$

For $p-n \leq j \leq p-1$,

$$
u_{j}:=v^{j-p+n+1} .
$$

Then above defined ordering $\left\{u_{0}, u_{1}, \ldots, u_{p-1}\right\}$ of vertices satisfies Theorem 2 . Step-4: Define $c: V\left(B_{n+1, k+1}\right) \rightarrow\{0,1,2, \ldots\}$ by $c\left(u_{0}\right)=0$ and $c\left(u_{i+1}\right)=c\left(u_{i}\right)+$ $p-1-L\left(u_{i}\right)-L\left(u_{i+1}\right)-\omega+1,0 \leq i \leq p-1$.

Output: The span of $c$ is $c\left(u_{p-1}\right)=h c(G)=c\left(u_{0}\right)+(p-1)^{2}-2\left(\sum_{u \in V(G)} \mathcal{L}(u)\right)-$ $(p-1)(n-1)=(p-1)^{2}-2 \mathcal{L}(G)-(p-1)(n-1)$ which is exactly the right-hand side of (8) by using (9) and (10) in the case of symmetric block graphs.

Thus, from Case - 1 and Case - 2, we obtain $h c\left(B_{n+1, k+1}(d)\right)$

$$
= \begin{cases}n^{2}(k+1)\left[\Phi_{r}(k n)\left((k+1) \Phi_{r}(k n)-2 r\right)+\frac{2\left(\Phi_{r}(k n)-r\right)}{k n-1}\right]+n, & \text { if } d \text { is even, } \\ k n^{2}(n+1)\left[\Phi_{r}(k n)\left(k(n+1) \Phi_{r}(k n)-2 r+1\right)+\frac{2\left(\Phi_{r}(k n)-r\right)}{k n-1}\right], & \text { if } d \text { is odd }\end{cases}
$$

Example 1. An optimal hamiltonian coloring of $B_{4,2}(4)$ using the procedure of Theorem 7 is shown in Figure 1 -(a).

For $B_{4,2}(4), k=1, n=3, d=4, r=\left\lfloor\frac{d}{2}\right\rfloor=2$ and $\Phi_{\left\lfloor\frac{d}{2}\right\rfloor}(k n)=\Phi_{2}(3)=1+3$ $=4$. By Theorem $7 h c\left(B_{4,2}(4)\right)$ 
$=n^{2}(k+1)\left[\Phi_{\left\lfloor\frac{d}{2}\right\rfloor}(k n)\left((k+1) \Phi_{\left\lfloor\frac{d}{2}\right\rfloor}(k n)-2\left\lfloor\frac{d}{2}\right\rfloor\right)+\frac{2\left(\Phi_{\left\lfloor\frac{d}{2}\right\rfloor}(k n)-\left\lfloor\frac{d}{2}\right\rfloor\right)}{k n-1}\right]+n$

$=3^{2} \cdot(1+1)\left[4((1+1) \cdot 4-2 \cdot 2)+\frac{2(4-2)}{3-1}\right]+3=327$.

Example 2. An optimal hamiltonian coloring of $B_{4,2}(5)$ using the procedure of Theorem 7 is shown Figure 1 -(b).

For $B_{4,2}(5), k=1, n=3, d=5, r=\left\lfloor\frac{d}{2}\right\rfloor=2$ and $\Phi_{\left\lfloor\frac{d}{2}\right\rfloor}(k n)=\Phi_{2}(3)=1+3$ $=4$. By Theorem $7 h c\left(B_{4,2}(4)\right)$

$=k n^{2}(n+1)\left[\Phi_{\left\lfloor\frac{d}{2}\right\rfloor}(k n)\left(k(n+1) \Phi_{\left\lfloor\frac{d}{2}\right\rfloor}(k n)-2\left\lfloor\frac{d}{2}\right\rfloor+1\right)+\frac{2\left(\Phi_{\left\lfloor\frac{d}{2}\right\rfloor}(k n)-\left\lfloor\frac{d}{2}\right\rfloor\right)}{k n-1}\right]$ $=1 \cdot 3^{2} \cdot(3+1)\left[4(1 \cdot(3+1) \cdot 4-2 \cdot 2+1)+\frac{2(4-2)}{3-1}\right]=1944$.

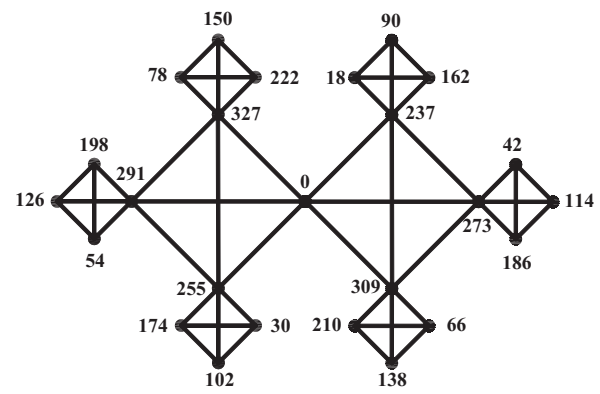

(a)

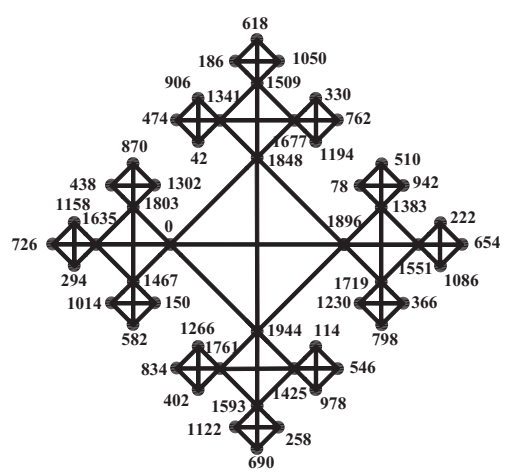

(b)

Fig. 1. Optimal hamiltonian coloring of $B_{4,2}(4)$ and $B_{4,2}(5)$.

\section{References}

1. Bantva, D.: On hamiltonian colorings of trees, communicated.

2. Chartrand, G., Nebeský, L., Zhang, P.: Hamiltonian coloring of graphs, Discrete Applied Math., 146, 257-272, (2005).

3. Chartrand, G., Nebeský, L., Zhang, P.: On hamiltonian colorings of graphs, Discrete Math., 290, 133-143, (2005). 
4. Chartrand, G., Escuadro, H., Zhang, P.: Detour distance in graphs, J. Combin. Math. Combin. Comput., 53, 75-94, (2005).

5. Khennoufa, R., Togni, O.: A note on radio antipodal colourings of paths, Mathematica Bohemica, 130(3), 277-282, (2005).

6. Shen, Y., He, W., Li, X., He, D., Yang, X.: On hamiltonian colorings for some graphs, Discrete Applied Math., 156, 3028-3034, (2008).

7. Vaidya, S. K., Bantva, D. D.: Symmetric regular cacti - properties and enumeration, Proyecciones Journal of Mathematics, 31(3), 261-275, (2012).

8. West, D. B.: Introduction to Graph theory, Prentice-Hall of India, (2001). 